\title{
Research Paper \\ Effect of Health-promoting Lifestyle and Psychological Well-being on Anxiety Induced by Coronavirus Disease 2019 in Non-medical Students
}

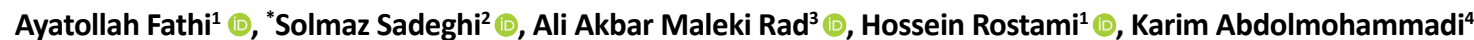

1. Institute of Law Enforcement and Social Studies, Tehran, Iran.

2. Department of Psychology, Institute of Charkh Niloofari, Tabriz, Iran.

3. Department of Biology, Faculty of Scince, Payam-e-Nour University, Tehran, Iran.

4. Department of Psychology, Faculty of Psychology and Educational Sciences, Payam-e-Nour University, Tehran, Iran.

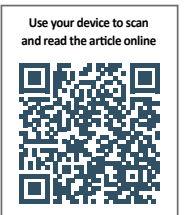

Cittation:Fathi A, Sadeghi S, Maleki Rad AA, Rostami H, Abdolmohammadi K. [Effect of Health-promoting Lifestyle and Psychological Well-being on Anxiety Induced by Coronavirus Disease 2019 in Non-medical Students (Persian)]. Journal of Arak University of Medical Sciences (JAMS). 2020; 23(5):698-709. https://doi.org/10.32598/JAMS.23.COV.1889.2

doi) https://doi.org/10.32598/JAMS.23.COV.1889.2

Key words:

Anxiety, Coronavirus, Lifestyle, Health, Psychological well-being

\section{A B STRACT}

Article Info:

Received:14 Apr 2020

Accepted:06 Jul 2020

Available Online:01 Dec 2020

\section{Extended Abstract}

\section{Introduction}

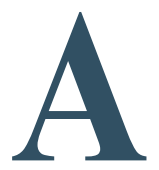

nxiety is one of the clinical signs of viral diseases such as coronavirus disease 2019 (COVID-19), which can weaken the immune system and make them vulnerable to such diseases [5]. Many studies have shown that stressful life events can affect psychological well-being [12-15, 18], and consequently lifestyles of people. The aim of this study is to identify the effects of health-promoting lifestyle and psychological well-being on the COVID-19-induced anxiety in non-medical students.

\section{Materials and Methods}

This is an analytical study. The study population includes all undergraduate students of Islamic Azad University in

\section{Corresponding Author:}

Solmaz Sadeghi

Address: Department of Psychology, Institute of Charkh Niloofari, Tabriz, Iran.

Tel: +98 (41) 33368864

E-mail: soli.sdgh@gmail.com 
Tabriz, Iran in 2020. After determining sample size using Krejcie \& Morgan table, 327 non-medical students were selected voluntarily. The data collection tools were Corona Disease Anxiety Scale developed by Alipour et al. [5]. Ryff's Scales of psychological Well- Being (SPWB) [28], (Containing six subscales of autonomy, environmental mastery, personal growth, purpose in life, positive relations with others and self-acceptance), and Health-Promoting Lifestyle Profile (HPLP) developed by Walker et al. [25] (Containing six subscales of responsibility, spiritual growth, physical activity, interpersonal relationships, nutrition, and stress management) which were completed by students online to due restrictions during the pandemic. After collecting questionnaires, 20 were excluded due to large amounts of outliers and 307 were included in the analysis. The statistical analysis was conducted in SPSS V. 16 using Stepwise regression method.

\section{Results}

Participants were 307 non-medical students; 256 females $(83.4 \%)$ and $51(16.6 \%)$ males with a mean age of 27 years. The stepwise regression analysis was used to evaluate the effects of SPWB dimensions on the COVID-19-induced anxiety. In the first step, the "personal growth" dimension entered the regression model, due to having the highest correlation coefficient with the dependent variable, and explained $71 \%$ of the variance in the anxiety. In the next step, the dimension of "positive relations with others" was entered into the model; this dimension together with the personal growth dimension explained $72 \%$ of the anxiety variance (Table 1). Other SPWB dimensions could not predict the COVID-19-induced anxiety and were then excluded from the regression model.

The stepwise regression analysis was also used to evaluate the effects of HPLP dimensions on the COVID-19-induced anxiety. In the first step, the "spiritual growth" dimension entered the regression model, due to having the highest correlation coefficient with the dependent variable, and explained $3 \%$ of the anxiety variance. In the next step, the dimension of "responsibility" was entered into the model; this dimension together with the spiritual growth dimension explained 9\% of the anxiety variance (Table 2). Other HPLP dimensions were excluded from the regression model.

\section{Discussion and Conclusion}

Findings from the present study showed that the two SPWB components of personal growth and positive relations with others together could explain $72 \%$ of the variance in the COVID-19-induced anxiety, and they had a significant negative relationship with the COVID-19-induced anxiety. This is consistent with the findings of some other studies [12-15, 18]. Personal growth means having a sense of continued development and, sense of realizing self potentials, and having more self-knowledge and effectiveness. Positive relations with others indicate having warm, satisfying, and trusting relationships with others [12]. Anxiety causes destructive effects on a person's physical and mental condition, and this in itself creates a vicious link between anxiety and the psychological well-being of the person; i.e.

Table 1. Regression coefficients for examining the effect of SPWB dimensions on the COVID-19-induced anxiety

\begin{tabular}{ccccccccc}
\hline Model & Variable & $\mathbf{B}$ & Std. error & $\boldsymbol{\beta}$ & $\mathbf{t}$ & $\mathbf{S i g}$. & $\mathbf{R}$ & $\mathbf{R}^{\mathbf{2}}$ \\
\hline 1 & Personal growth & -4.30 & 0.156 & -0.846 & -27.69 & 0.001 & 0.84 & 0.71 \\
& Personal growth & -4.23 & 0.158 & -0.831 & -26.70 & 0.001 & 0.84 & 0.72 \\
\hline & Positive relations with others & -0.350 & 0.153 & -0.071 & -2.29 & 0.023 & & \\
\hline
\end{tabular}
Journal of
Arak University of Medical Sciences

Table 2. Regression coefficients for examining the effect of HPLP dimensions on the COVID-19-induced anxiety

\begin{tabular}{ccccccccc}
\hline Model & Variable & $\mathbf{B}$ & Std. error & $\boldsymbol{\beta}$ & $\mathbf{t}$ & $\mathbf{S i g}$ & $\mathbf{R}$ & $\mathbf{R}^{2}$ \\
\hline 1 & Spiritual growth & -0.325 & 0.098 & -0.186 & -3.30 & 0.001 & 0.18 & 0.03 \\
\multirow{2}{*}{2} & Spiritual growth & -0.629 & 0.118 & -0.361 & -5.31 & 0.001 & 0.30 & 0.09 \\
& Responsibility & -0.445 & 0.102 & -0.295 & -4.35 & 0.001 & & \\
\hline
\end{tabular}


anxiety endangers his/her psychological well-being [14]. Another finding of this study showed that people who had higher spiritual growth and responsibility (two HPLP components ) had less COVID-19-induced anxiety, indicating a significant negative relationship between them, which is consistent with other studies [3, 23, 26, 33]. Spiritual growth is an internal process to break old habits and ideas, misconceptions and false beliefs about life [3, 33]. Responsibility makes a person sensitive and responsible to maintain and improve self health by visiting a doctor in case of need; hence, they report less anxiety [26].

\section{Ethical Considerations}

\section{Compliance with ethical guidelines}

This study was approved by University of Tabriz (Code:IR.TABRIZU.REC.1399.018). All ethical principles are considered in this article. The participants were informed about the purpose of the research and its implementation stages. They were also assured about the confidentiality of their information and were free to leave the study whenever they wished, and if desired, the research results would be available to them.

\section{Funding}

This research did not receive any grant from funagencies in the public, commercial, or non-profit sector.

\section{Authors' contributions}

Project design, guidance, editing theoretical foundations, statistical analysis and scientific editing of the article: Ayatollah Fathi; Compilation of proposal, writing of the introduction, statistical analysis and participation in writing theoretical foundations and articles: Solmaz Sadeghi; Participation in project design, discussion and writing of results and article: Ali Akbar Maleki Rad; Guiding and editing theoretical foundations, participation in data collection and analysis: Hossein Rostami; Participation in data collection and analysis: Karim Abdolmohammadi.

\section{Conflicts of interest}

The authors declare no conflict of interest. 


\section{مقاله يخوهشى \\ تأثير ابعاد سبك زندكَ ارتقادهنده سلامت و بهزيستى روانشناختى در اضطراب كرونا (كوويد

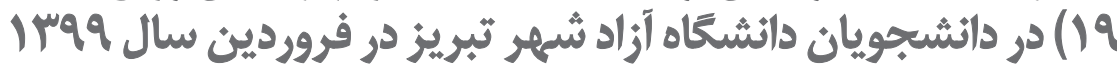

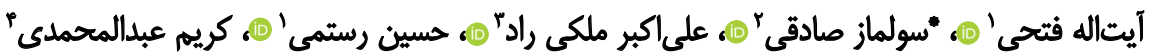 \\ ا. ئروهشعاه علوم انتظامى ومطالعات اجتماعى ناجا، تهران، ايران.

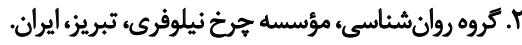

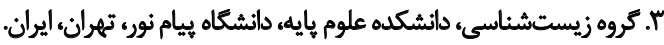

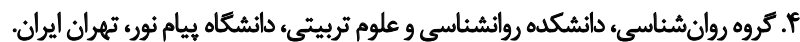

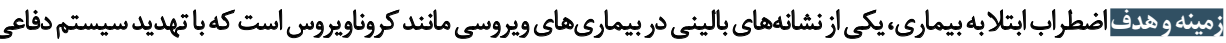

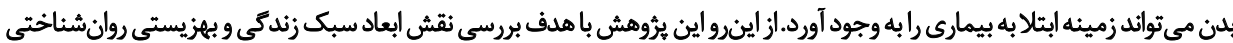

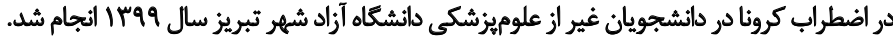

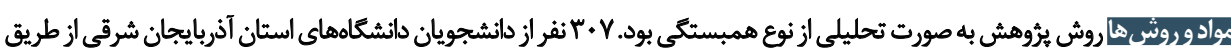

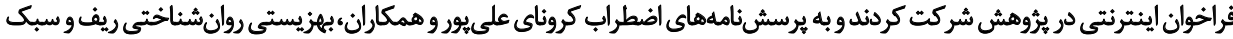

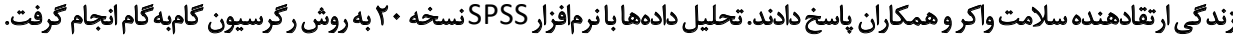
ملاحظات اخلاقى اين بثروهش مورد تاييد كميته اخلاق دانشكاه تبريز قرار كرفته است (IR.TABRIZU.REC.1399.018).

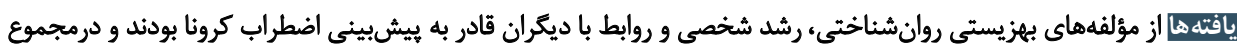

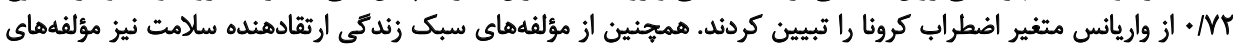

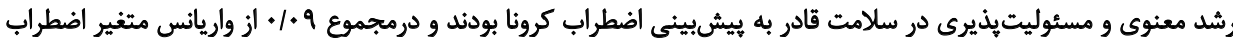
كرونا را تبيين كردند.

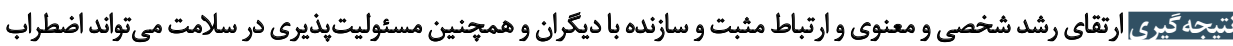

از اين عوامل اضطراب است. اضطراب در زندكى امروزى كه بسيار

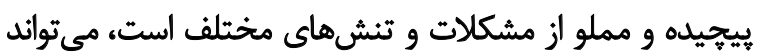

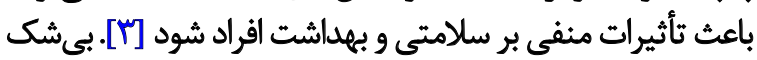

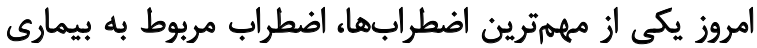
نيروى انسانى مهمثرين سرمايه جامعه است و هرجها اين سرمايه

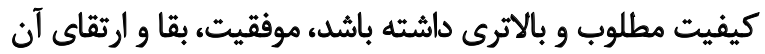

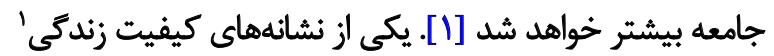

كروناويروسها (كوويد 9 1)" خانواده بزركى از ويروسها هستند كه ممكن است باعث عفونتهاى تنفسى أز سرماخورديى آنى

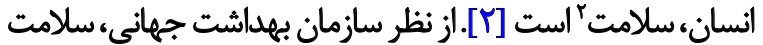

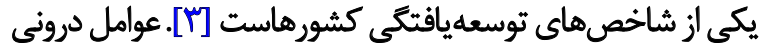

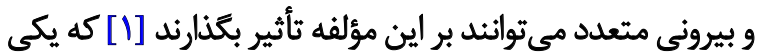

\section{COVID-19}

1. Quality of life

2. Health 
نشان دادهاند افراد مبتلا به بيمارى هاى زمينهاى در معرض خطري

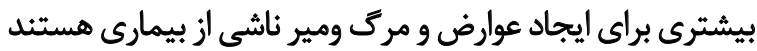

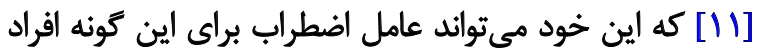

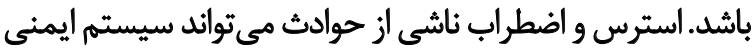

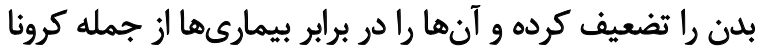

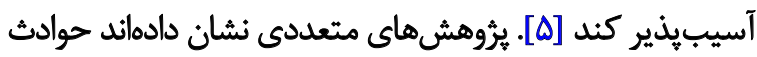

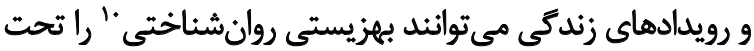

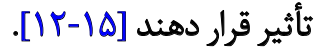

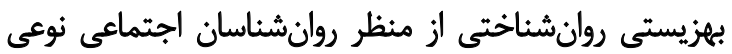

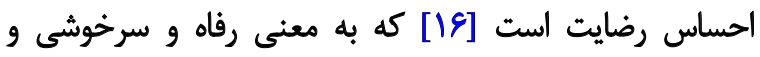

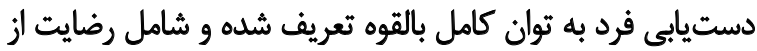

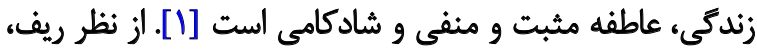

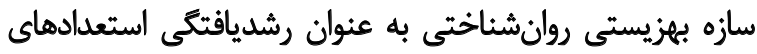

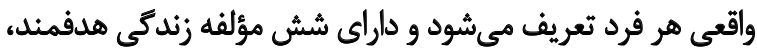

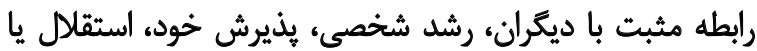

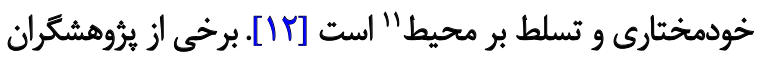

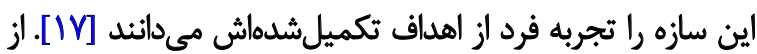

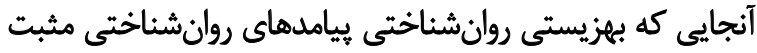

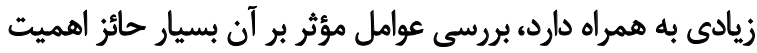

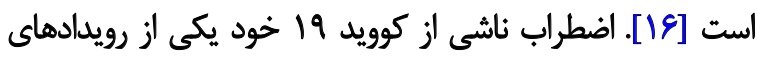

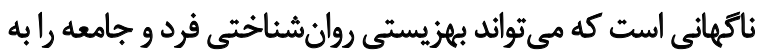

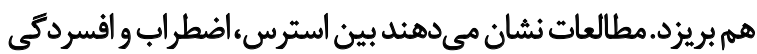

و بهزيستى روانشناختى رابطه معنى دارى وجود دارد [1/1)]. در دنياى كنونى سلامت به عنوان دستاوردى مهرم تلقي

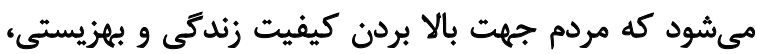

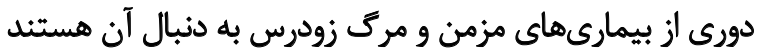

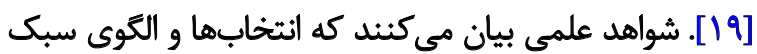

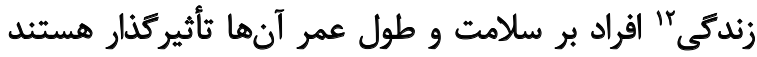

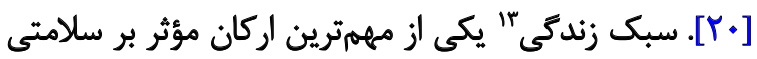

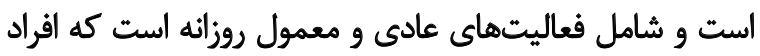

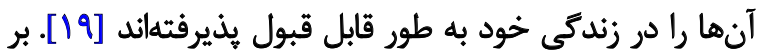

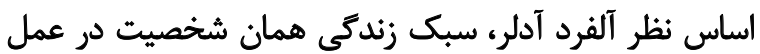

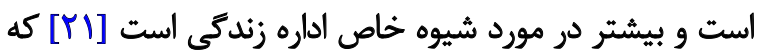

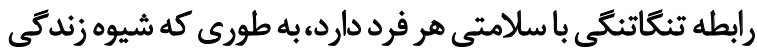

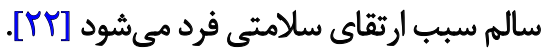

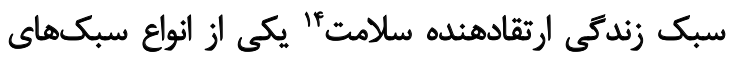

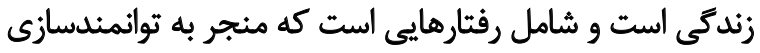

10. Psychological well-being

11. Purpose in life, positive relations with others, personal growth, self-acceptance, autonomy, mastering the environment 12. Lifestyle pattern

13. Lifestyle

14. Healthy lifestyle enhancer
كرفته تا بيمارى هاى شديدتر مانند مرسه و سارس " شوند [ه]].

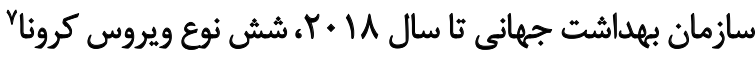

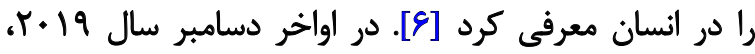

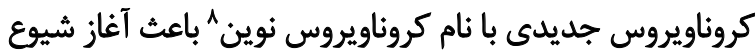

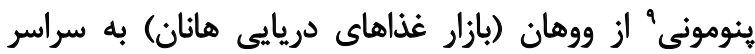

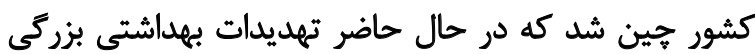

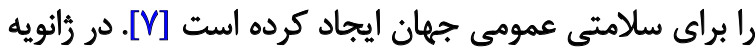

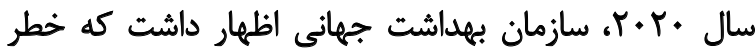

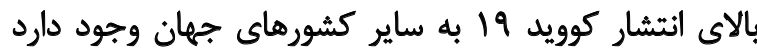

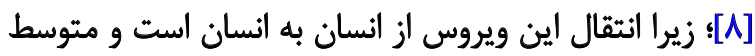

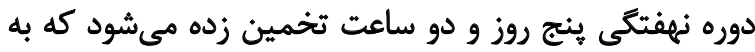

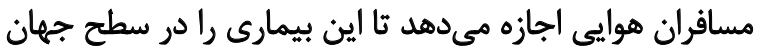

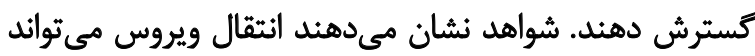

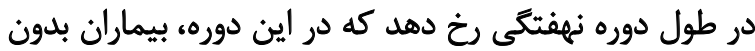

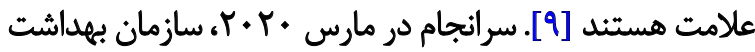

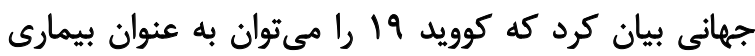

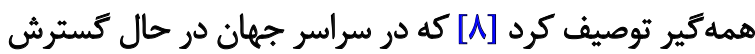

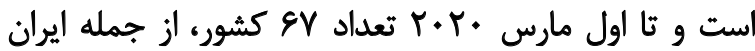

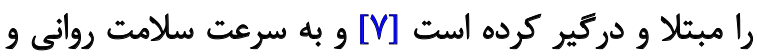
جسمى را به خطر انداخته است [هرد].

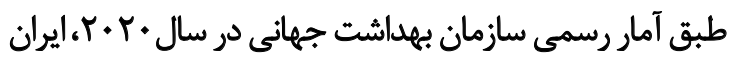

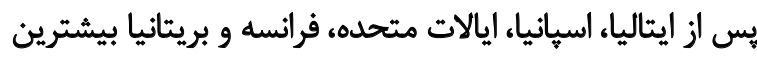

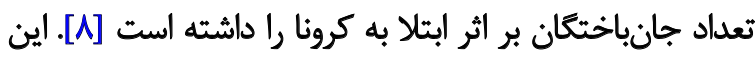

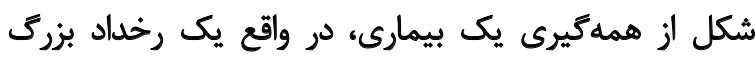

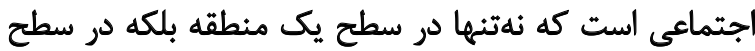

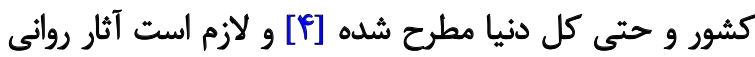

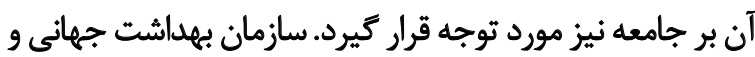

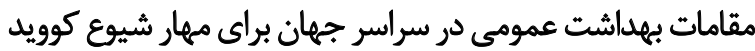

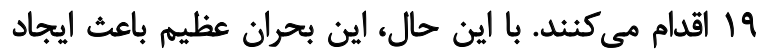

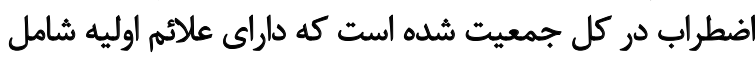

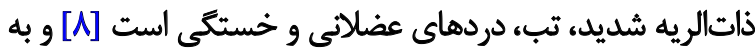

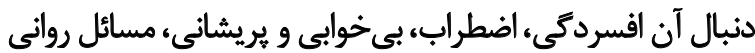

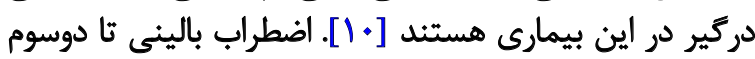

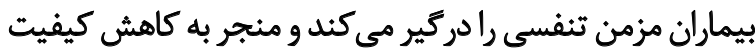

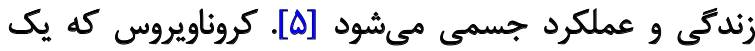

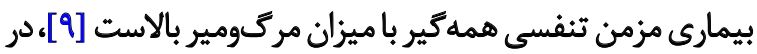

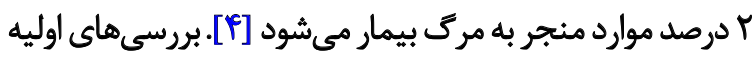

\section{MERS}

6. SARS

7. 1. HCoV229-E, 2. HCoV-OC43, 3. SARS-CoV, 4. HCoV-NL63, New Haven coronavirus, 5. HumancoronavirusHKU1, 6. MERS-CoV 8. Severe acute respiratory syndrome coronavirus2) SARS-CoV2(or-2019nCoVor"novel coronavirus 2019)

9. Pneumonia 


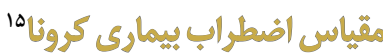

اين يرسشنامه با هدف تعيين ميزان اضطراب كرونا در نمونه

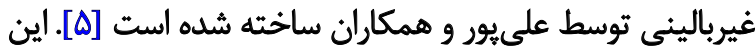

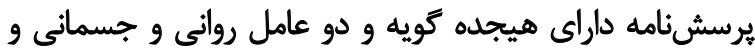

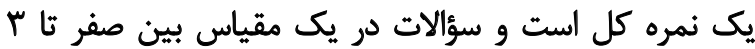

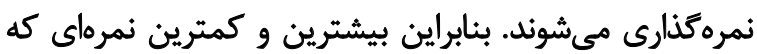

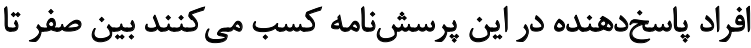

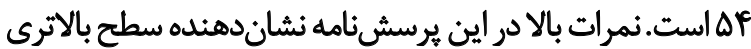

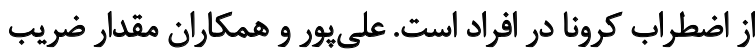

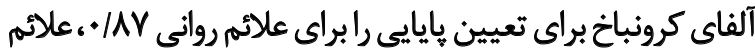

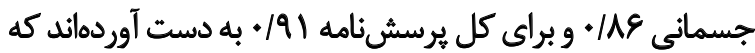

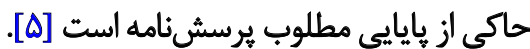

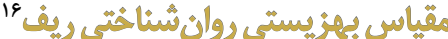

اين برسشنامه توسط ريف در سال 1919 و با هدف

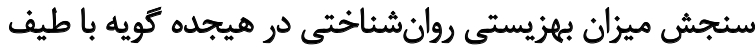

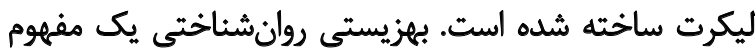

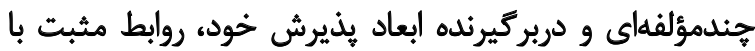

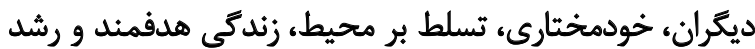

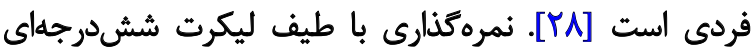

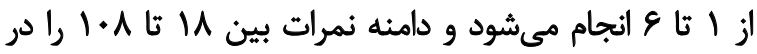

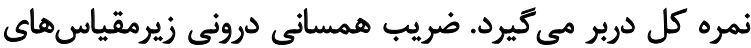

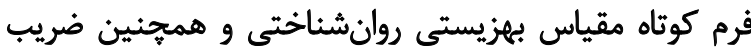

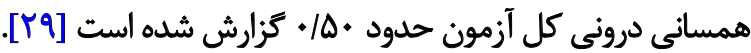

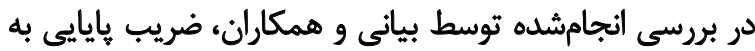

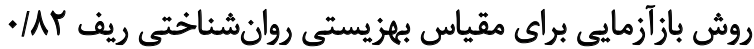

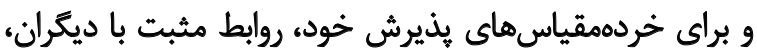

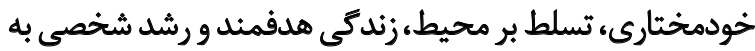

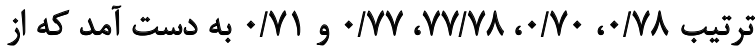

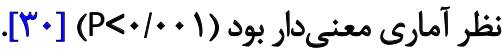

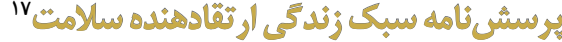

يرسشنامه سبك زندكى ارتقادهنده سلامت را واكر و و وارئ

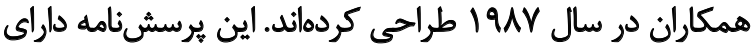

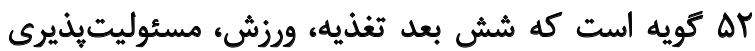

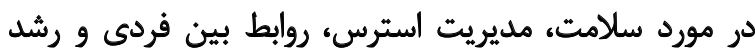

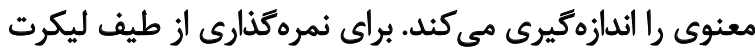

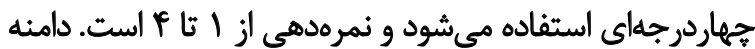

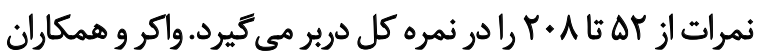

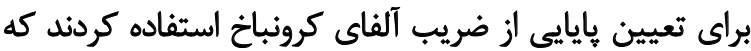

افراد براى افزٔايش كنترل بر سلامت خود ودرنهايت بهبود سلامت

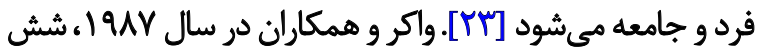

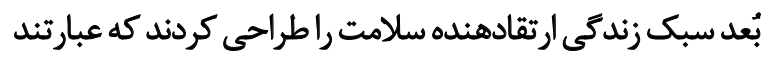

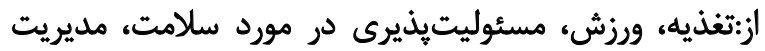

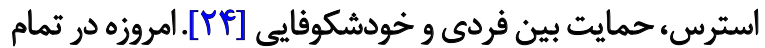

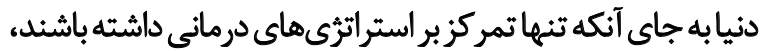

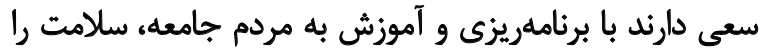

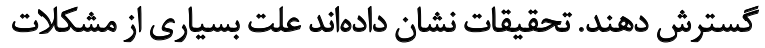

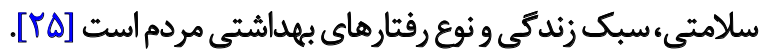

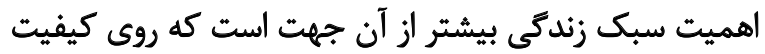

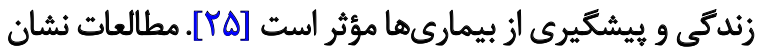

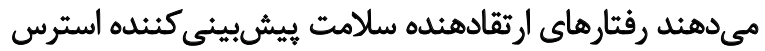

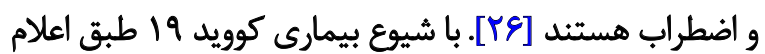

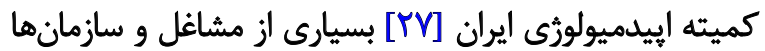

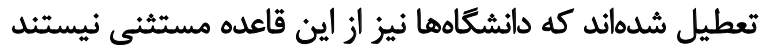

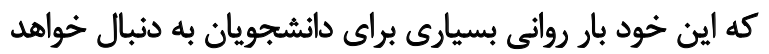

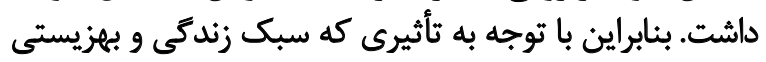

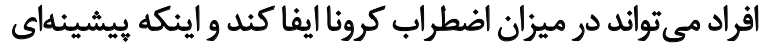

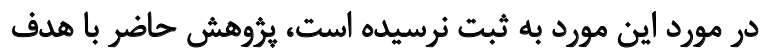

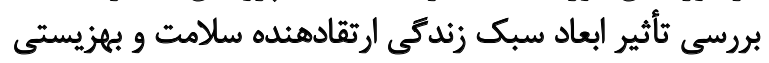

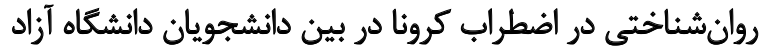

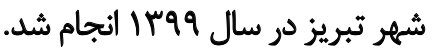

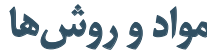

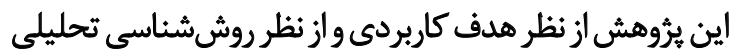

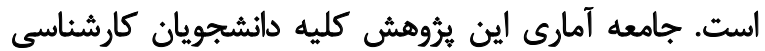

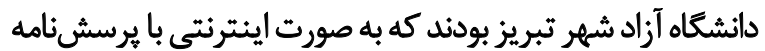

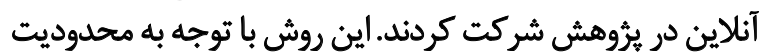

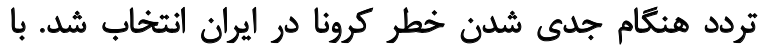

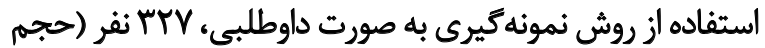

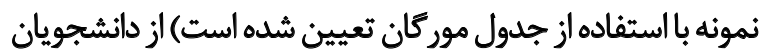

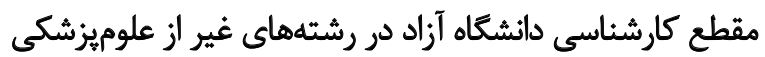

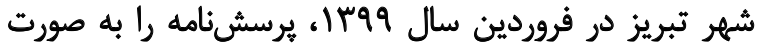

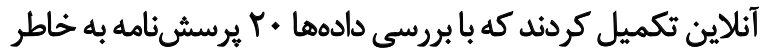

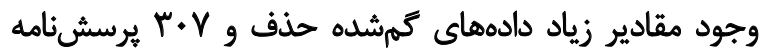

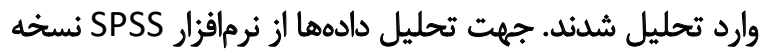

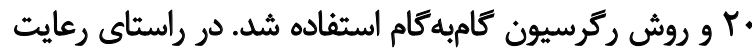

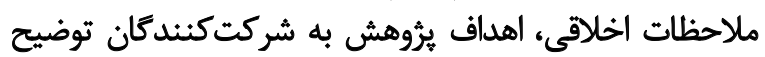

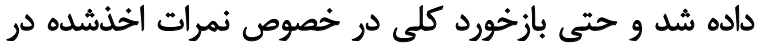
متغيرها به شركت كنئدكان داده شد. 
جدول ا. شاخصهاى توصيفى متغيرهاي بهزيستى روانشناختى، سبك زئدكى و اضطراب كرونا

\begin{tabular}{|c|c|c|c|c|c|c|}
\hline \multicolumn{2}{|c|}{ أزمون كولموكروف اسميرنوف } & \multirow{2}{*}{ حداكثر } & \multirow{2}{*}{ حداقل } & \multirow{2}{*}{ مياتكيندانحرافمعيار } & \multirow{2}{*}{ مثغيرها } & \\
\hline معنى دارى & آماره & & & & & \\
\hline - /AVP &.$/ 019$ & to & 8 & $1 . / 9 r \pm 1 / 9$. & خُودمخْتَارى & \\
\hline ./VAF &.$/ 14$ & 10 & $r$ & $1 . / 81 \pm 1 / \Delta 9$ & تسلط بر هحيط & $\ddot{\psi}$ \\
\hline.$/ 4 \lambda$ & $y / \cdot 9$ & ir & $r$ & $1 r / V \Delta \pm 1 / 99$ & رشد شخصى & : \\
\hline.$/ \Delta \Delta H$ &.$/ 9 \Delta$ & 10 & e & $N \Delta F \pm Y / \cdot \Delta$ & رابطه با ديكران & 3 \\
\hline.$/ 4 T$ & $1 / \%$ & if & $r$ & $N \& q \pm 1 / q$. & هلفمندى & \\
\hline$\cdot|8 A|$ & . Are & 10 & $ه$ & $1 . / 11 \pm 1 / \Delta 8$ & لئيرش خود & \\
\hline$\cdot / \mathrm{AV}$ & . $/ \Delta A N$ & rq & 1. & $r \Delta / r \Lambda \pm \Delta / \Lambda$. & ر رشد معنوى & \\
\hline.$|P A|$ & $\mid / M I$ & $p q$ & 10 & $r q / \wedge q \pm / M T$ & مستوليتئيرى & \\
\hline.$/ 118$ & $1 / 19$ & rA & $\wedge$ & WEVET/.V & روابط بين فردى & 急 \\
\hline ./Ms &.$/ 1 \Delta$ & 19 & ه & $11 / f+ \pm r / \Delta q$ & ملديريت استرس & 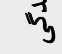 \\
\hline.$/ 11$ & ./NTA & ra & $\checkmark$ & IF/WEY/R & ورزش & \\
\hline.$|\Delta \Delta|$ & - (aro & ra & $A$ & $18 / 19 \pm+/ 11$ & تغذيه & \\
\hline.$/ F \Delta F$ & ./IIT & $\Delta r$ & - & $|F / r| \pm \mid+/ / r$ & اضطراب كرونا & \\
\hline
\end{tabular}

در كام بعدى متغير رابطه با ديكران به دليل دارا بودن بالاترين

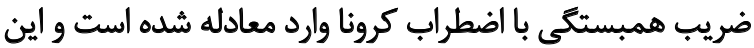

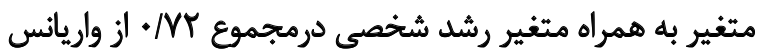

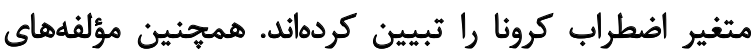

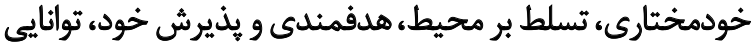

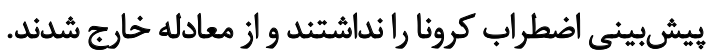

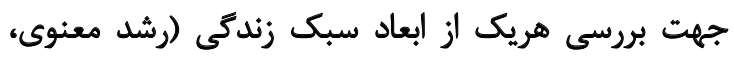

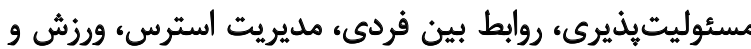

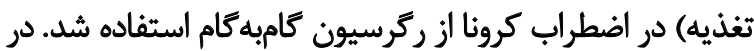

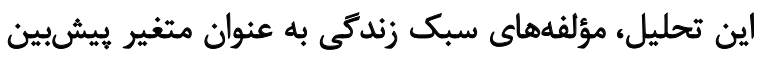

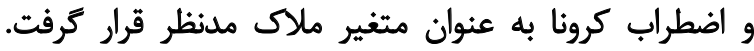

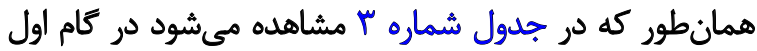

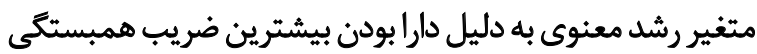

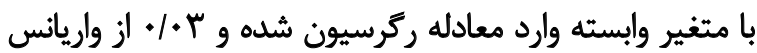

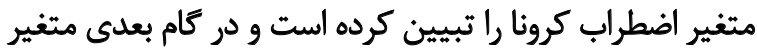

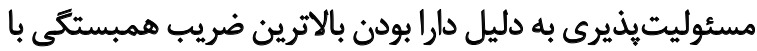

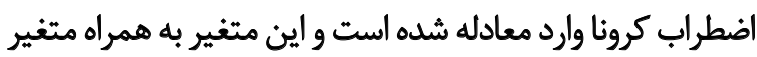

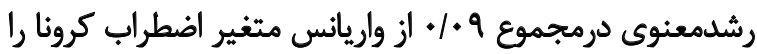

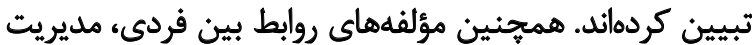
استرس، ورزش و تغذيه از معادله خارج شدند.

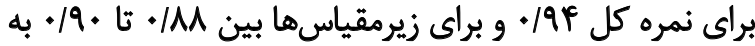

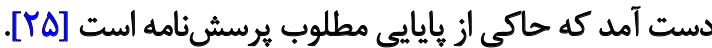

ياقتهها

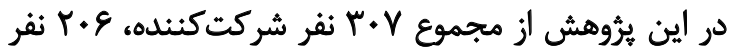

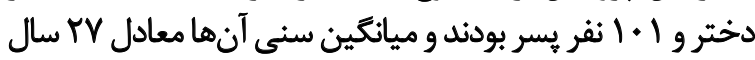

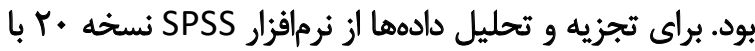

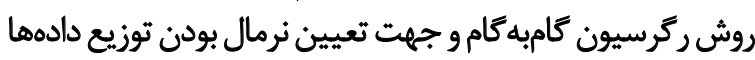

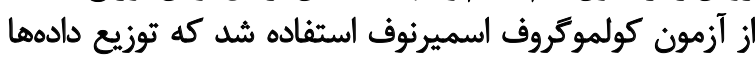

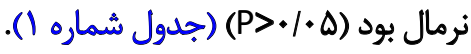

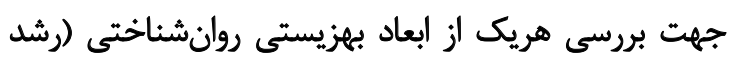

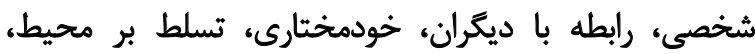

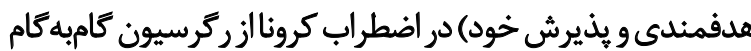

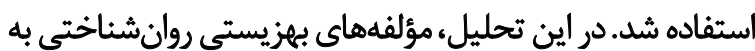

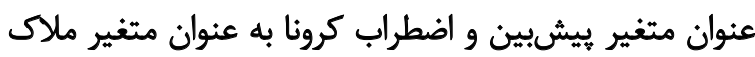

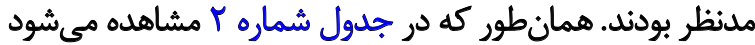

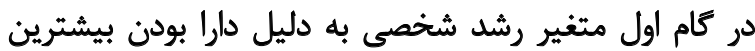

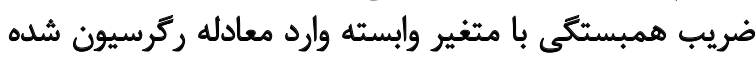

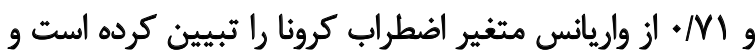


جدول r. نتايج ركرسيون كامبه كام در زميئه نقش ابعاد بهزيستى روانشئاختى در اضطراب كرونا

\begin{tabular}{|c|c|c|c|c|c|c|c|c|}
\hline $\mathbf{R}^{2}$ & $\mathbf{R}$ & Sig. & $\mathbf{t}$ & بتا & خطاى استاندارد & B & متغير & مدل \\
\hline$\cdot \mid M$ & - /AP & $.1+.1$ & $-r V / E q$ &.- ANE & $.11 \Delta 8$ & $-r / \pi$ & رشد شخصيى & 1 \\
\hline$\cdot / N$ & - /Ar & $\begin{array}{l}.+1 \\
.41\end{array}$ & $\begin{array}{l}-r g / V . \\
-T / T q\end{array}$ & $\begin{array}{l}\cdot-|A r| \\
-+|\cdot V|\end{array}$ & $\begin{array}{l}. / 1 \Delta A \\
. / N A T\end{array}$ & $\begin{array}{l}-r / \pi \\
-\pi \Delta .\end{array}$ & رشد شخصى رابطه با ديكران & $r$ \\
\hline
\end{tabular}

جدول ؟. نتايج ركرسيون كامبه كام در زميئه نقش ابعاد سبك زندكى در اضطراب كرونا

\begin{tabular}{|c|c|c|c|c|c|c|c|c|}
\hline $\mathbf{R}^{2}$ & $\mathbf{R}$ & Sig. & $\mathbf{t}$ & بتا & خُطاي استاندارد & B & متغير & مدل \\
\hline 1.4 &.$/ A$ & $.1 .+1$ & $-r / r$. &.$- / 118$ & .1 .41 &.$- / M T \Delta$ & رخد معنوى & 1 \\
\hline 1.9 & $+/ \mu$ & $\begin{array}{l}.1 .01 \\
.1 .+1\end{array}$ & $\begin{array}{l}-\Delta / \mu \Delta \\
-\varphi / \% \Delta\end{array}$ & 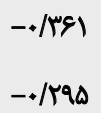 & $\begin{array}{l}. / 111 \\
. / 1 . r\end{array}$ & $\begin{array}{l}-. / 9499 \\
-. / 47 a\end{array}$ & رسئوليتهيذيرى معنوى & $r$ \\
\hline
\end{tabular}

sing

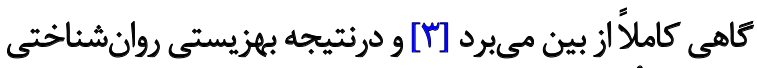

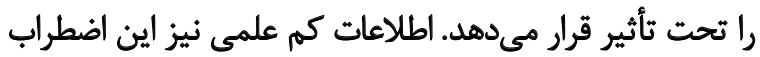

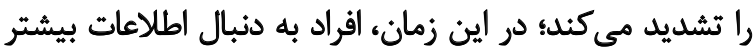

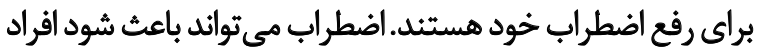

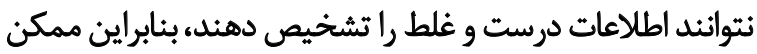

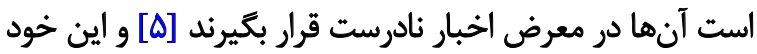

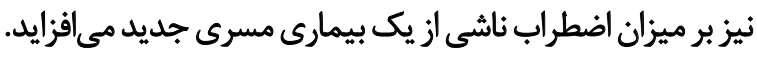

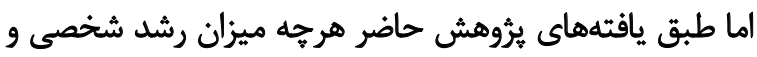

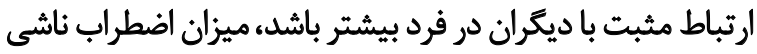

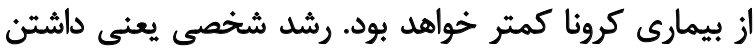

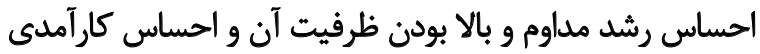

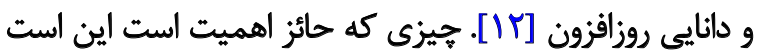

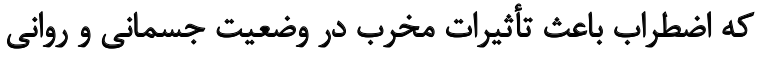

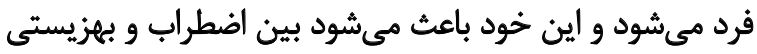

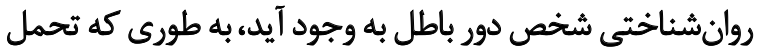

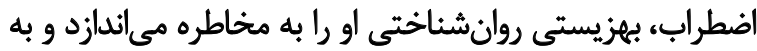

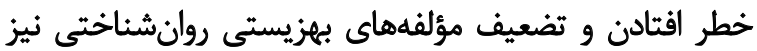

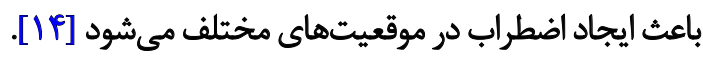

يافته ديكر بثروهش حاضر ارتباط معكوس رابطه با ديكران و

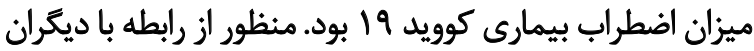

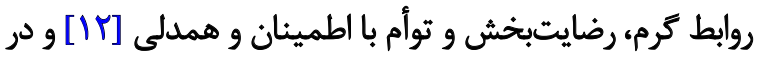

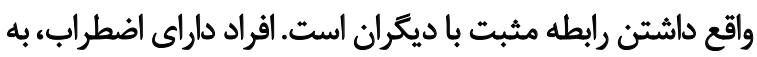

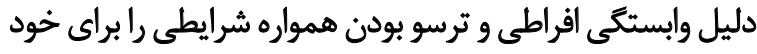

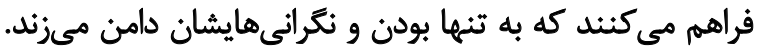

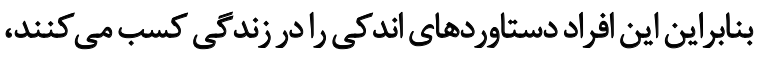

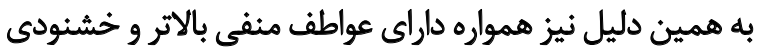

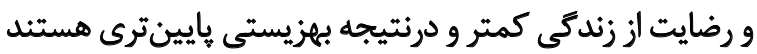

يافتهاى حاصل از يزوهش حاضر نشان مىدهند از بين

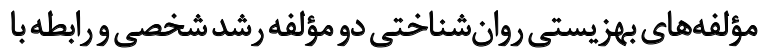

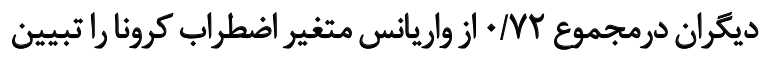

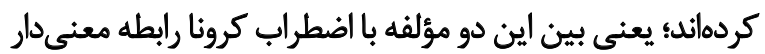

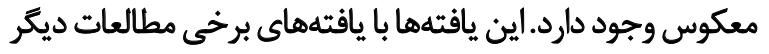

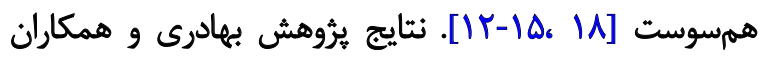

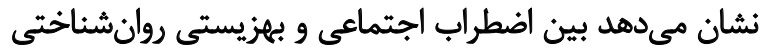

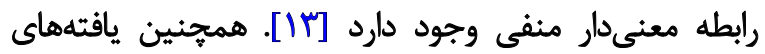

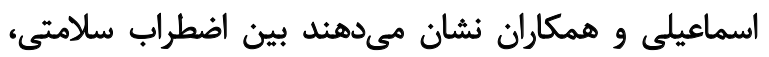

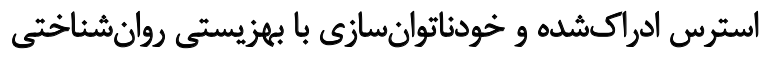

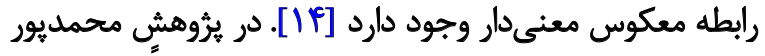

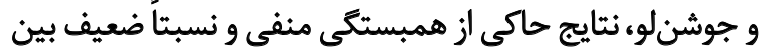

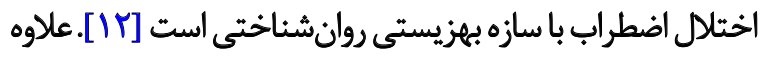

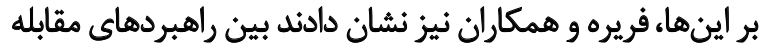

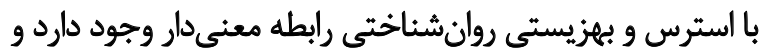

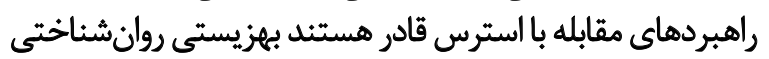

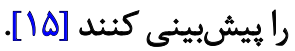
در تبيين نتايج فوق مى توان كفت اضطراب در زندكى انسان

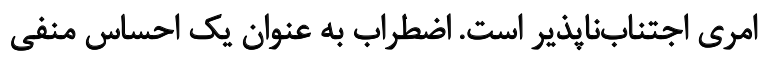

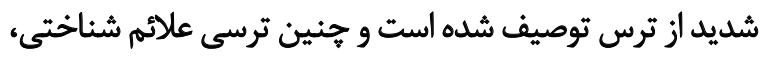

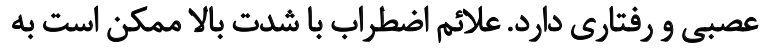

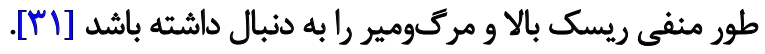

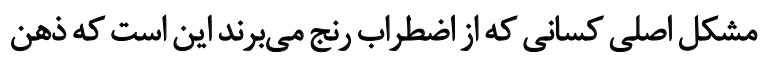

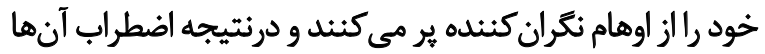

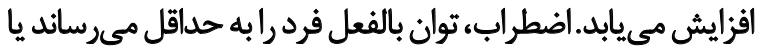


اضطراب با رشد معنوى بود [ب] ـ يُروهش فاطمى و همكاران نشارن

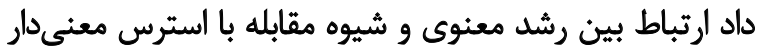

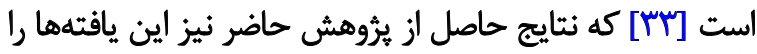

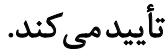

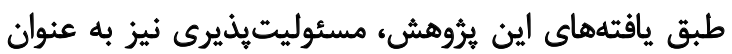

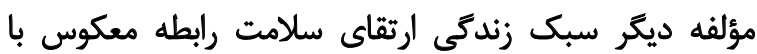

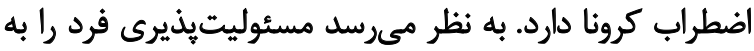

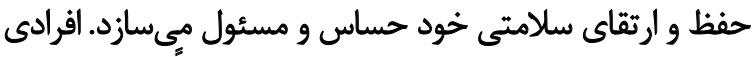

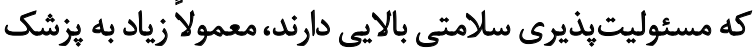

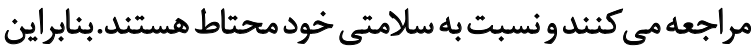

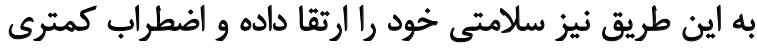

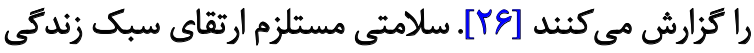

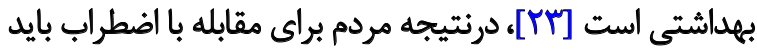

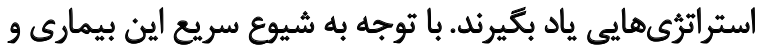

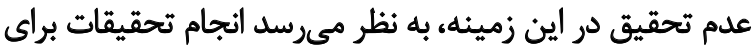

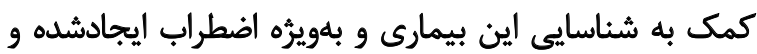

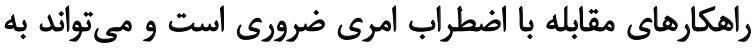

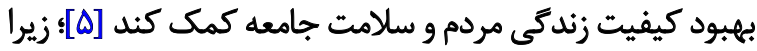

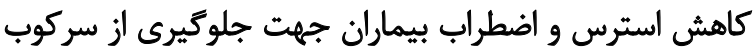

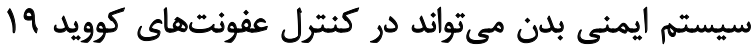

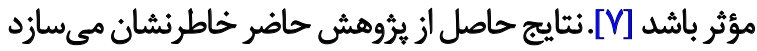

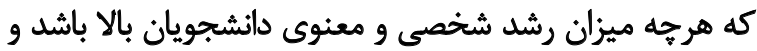

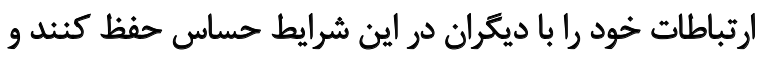

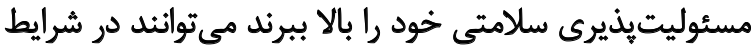

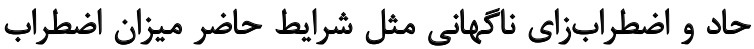
كمترى راتجربه كثيند.

\section{نتيجه تيرى}

با توجه به نتايج بهدست آمده مى توان نتيجه كرفت كمه ارتقاى

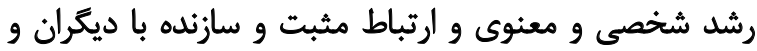

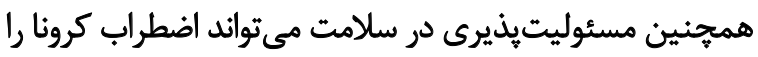

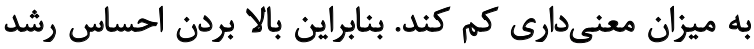

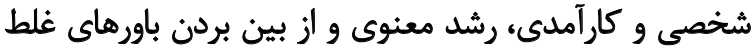

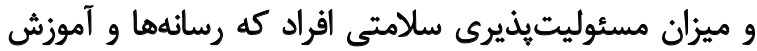

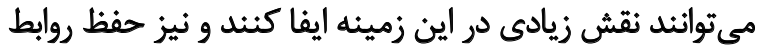

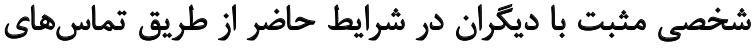

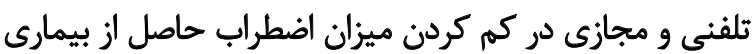

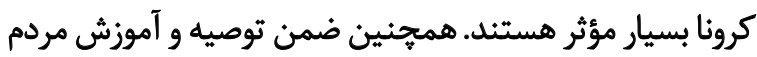

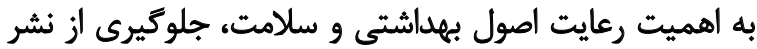

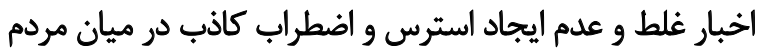
براى حفظ آرامش آنها نيز براى مقابله بارئ اين بيمارى از اهميت دانيت برزايى برخوردار است.

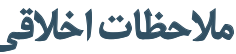

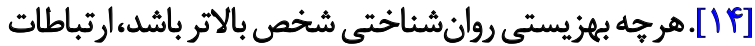

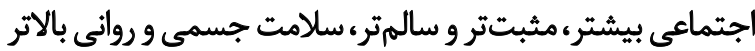

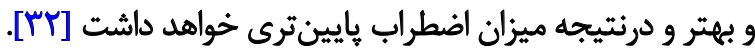

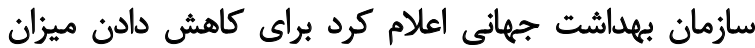

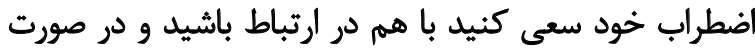

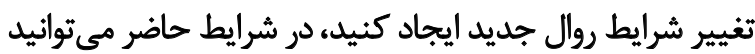

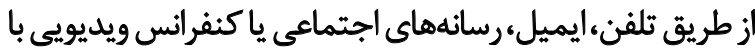
يكديكر در ارتباط باشيد [1].

همجنين يافته ديكر اين مطالعه نشان داد از بين مؤلفههاى

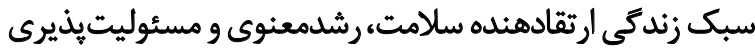

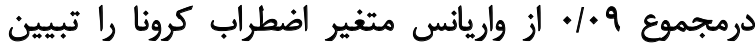

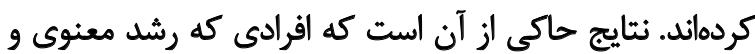

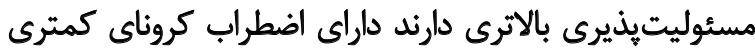

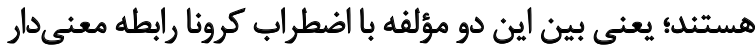

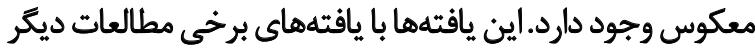

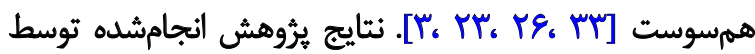

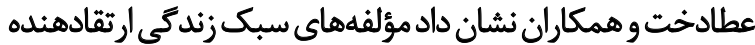

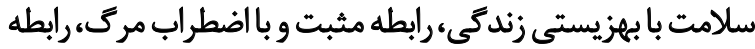

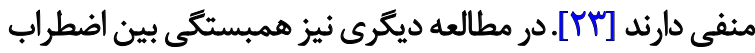

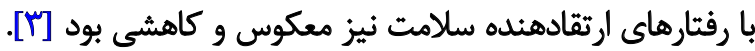

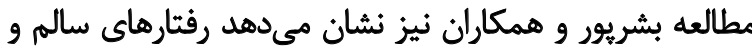

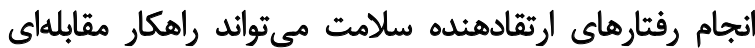

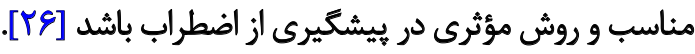
تدر تبيين نتايج فوق مى توان كفت ارتقاى سلامت، علم و هنر

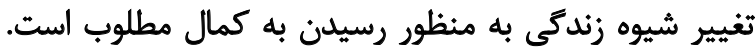

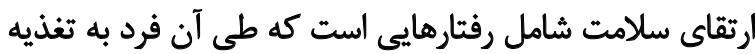

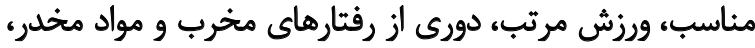

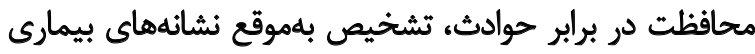
در بعد جسمى، كنترل عواطف و واحساسات و افكار و وكنار بـاري

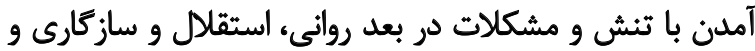

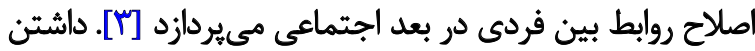

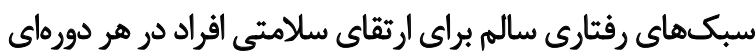

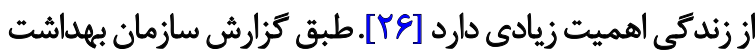

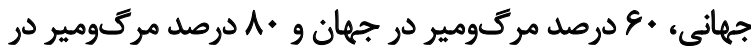

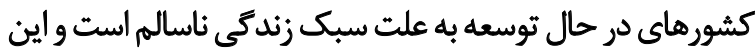

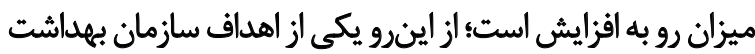

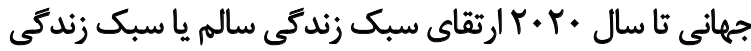
ارتقادهنده سلامت در افراد است [19 [19].

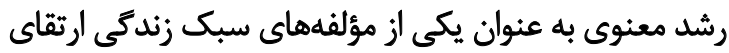

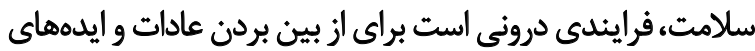

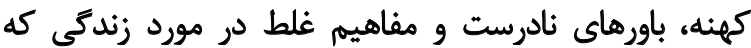
تحقيقات رابطه معنى دار معكوس وآن با اضطراب ران رانشان مى دمهند

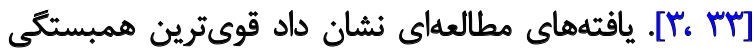




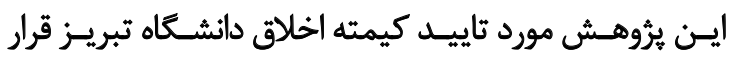

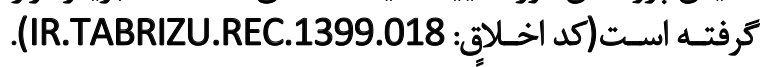

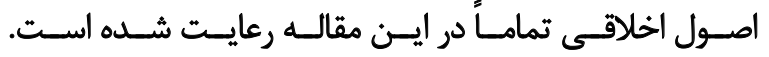

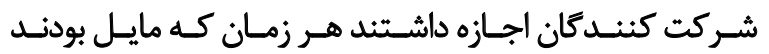

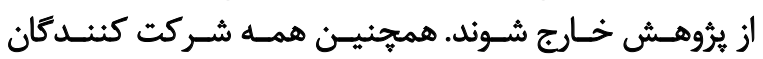

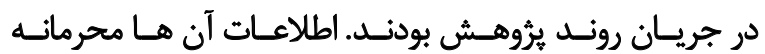

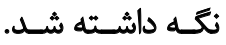

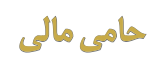

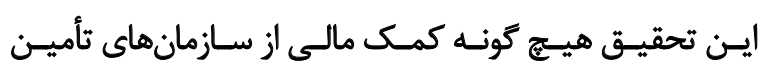

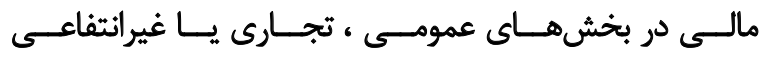
دريافـت نكـرد.

\section{هشار كت نويسند كان}

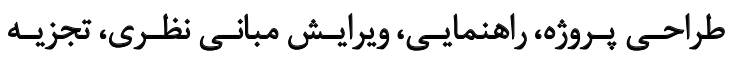

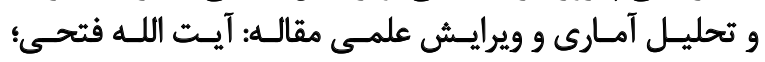

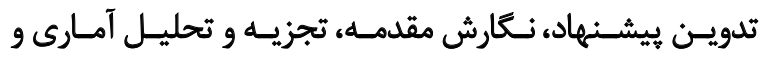

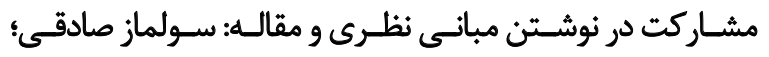

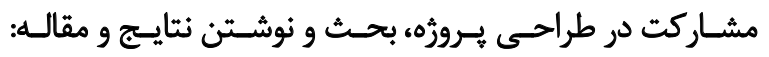

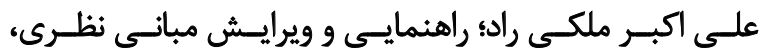

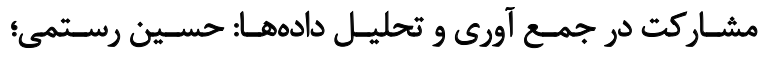

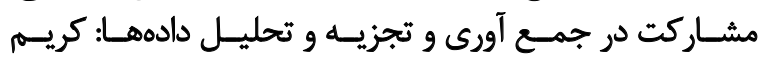

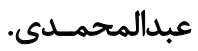

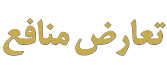
بنابر اظهار نويسندكان اين مقاله تعارض و منافع ندارد 


\section{References}

[1] Ghazavi Z, Zeighami R, Sarichello ME, Shahsavari S. [Effective training of cognitive cognitive regulation strategies on the psychological well-being of nurses working in psychiatric wards (Persian)]. Iran J Psychiatry. 2019; 7(4):64-73. http://ijpn.ir/article1-1406-fa.html

[2] Sharbatian MH, Imani N. [Sociological analysis of social health of youth and factors affecting it a case study:(youth 18 to 30 years old in Ghaen City) (Persian)]. Appl Sociol. 2019; 29(1):167-88. [DOI:10.22108/JAS.2017.75233.0]

[3] Norouzinia R, Aghabarari M, Kohan M, Karimi M. [Health promotion behaviors and its correlation with anxiety and some students' demographic factors of Alborz University of Medical Sciences (Persian)]. J Health Promot Manag. 2013; 2 (4) :39-49. http://jhpm.ir/article-1-236-en.html

[4] Alizadeh Fard S, Saffarinia M. [Predicting mental health based on anxiety and social correlation due to coronary heart disease (Persian)]. Soc Psychol Res. 2020; 9(36):129-41. http://www.socialpsychology.ir/article 105547.html

[5] Alipour A, Ghadami A, Alipour Z, Abdullahzadeh H. [Preliminary validation of Corona Anxiety Scale (CDAS) in the Iranian sample (Persian)]. J Health Psychol. 2020; 8(32):163-75. [DOI:10.30473/ HPJ.2020.52023.4756

[6] Corman VM, Muth D, Niemeyer D, Drosten C. Hosts and sources of endemic human coronaviruses. Adv Virus Res. 2018; 100:163188. [DOI:10.1016/bs.aivir.2018.01.001] [PMID] [PMCID]

[7] Farnoosh G, Alishiri G, Hosseini Zijoud S R, Dorostkar R, Jalali Farahani A. [Recognition of the new Coronavirus - 2019 and Cowide - 19 based on available evidence - review review (Persian)]. J Mil Med. 2020; 22(1):1-11. [DOI:10.30491/JMM.22.1.1]

[8] Liu N, Zhang F, Wei C, Jia Y, Shang Z, Sun L, et al. Prevalence and predictors of PTSS during COVID-19 outbreak in China hardest-hit areas: Gender differences matter. Psych Res. 2020; 287:112921. https://doi.org/10.1016/j.psychres.2020.112921

[9] Kooraki S, Hosseiny M, Myers L, Gholamrezanezhad A. Coronavirus outbreak:What the department of radiology should know. J Am Coll Radiol. 2020; 17(4):447-51. [DOI:10.1016/j. jacr.2020.02.008] [PMID] [PMCID]

[10] Lai J, Ma S, Wang Y, Cai Z, Hu J, Wei N, et al. Factors associated with mental health outcomes among health care workers exposed to coronavirus disease 2019. JAMA Netw Open. 2020; 3(3):e203976. [DOI:10.1001/jamanetworkopen.2020.3976] [PMID] [PMCID]

[11] Tavakoli A, Vahdat K, Keshavarz M. [Novel coronavirus disease 2019 (COVID-19):An emerging infectious disease in the 21st century (Persian)]. ISMJ. 2020; 22(6):432-50. [DOI:10.29252/ ismj.22.6.432]

[12] Mohammadpour S, Jooshanlou M. [Relationship between published anxiety disorder and obsessive-compulsive personality disorder with psychological well-being of students of Lorestan University of Medical Sciences in 2014:The mediating role of passionate obsessive love (Persian)]. J Rafsanjan Univ Med Sci. 2015; 14(5):353-66. https://journal.rums.ac.ir/browse.php?a_ id $=2275 \&$ slc lang=fa\&sid=fa\&ftxt=1\&html=1

[13] Bahadori Khosroshahi J, Hashemi Nosrat Abad TT. [The relationship between social anxiety, optimism and self-efficacy with psychological well-being in students (Persian)]. Stud Med Sci. 2012; 23(2):115-22. http://umj.umsu.ac.ir/article-1-1321-fa.htm
[14] Esmaeili S, Ghanbari Panah A, KoochakEntezar R. [Prediction of psychological well-being based on perceived health anxiety and stress with its mediating role of disability in married women working in the School of Nursing and Midwifery, Tehran University of Medical Sciences in 2018 (Persian)]. Iran J Nurs Res. 2020 14(6):46-52. http://ijnr.ir/article-1-2320-fa.html

[15] Freire C, Ferradás MDM, Valle A, Núñez JC, Vallejo G. Profiles of psychological well-being and coping strategies among university students. Front Psychol. 2016; 7:1554. [DOI:10.3389/fpsyg.2016.01554]

[16] Hatefnia F, Dar Taj F, Alipour A, Farokhi NA. [The effect of academic procrastination on psychological well-being:the mediating role of floating, positive-negative emotions and life satisfaction (Persian)]. Soc Psychol Res. 2019; 9(33):53-76. http://www.socialpsychology.ir/article_91536.htm

[17] Roslan S, Ahmad N, Nabilla N, Ghiami Z. Psychological Well-being among Postgraduate Students. Acta Medica Bulgarica. 2017; 44(1):35-41. [DOI:10.1515/amb-2017-0006]

[18] Sehati Yazdi A, Talebian Sharif J. [The relationship between Internet addiction and mental disorders (anxiety, stress and depression) and psychological well-being (Persian)]. J Mod Ideas Psychol. 2019; 2(6):1-9. http://jnip.ir/article-1-189-fa.html

[19] Najjar Nasab S, Dasht-e Bozorgi Z. [The effect of motivational interviews on lifestyle enhancing the health and blood pressure of women with eating disorder (Persian)]. J Health Psychol. 2019; 8(31):103-18. [DOI:10.30473/HPJ.2019.40367.4005]

[20] Mohammadi Zaidi I, Pakpour Haji Agha A, Mohammadi Zaidi B. [The validity and reliability of the Persian version of the Health Promotion Lifestyle Questionnaire (Persian)]. J Mazand Univ Med Sci. 2012; 22(Supple 1):103-13. http://jmums.mazums.ac.ir/browse.php?a_code=A10-377-4\&slc_lang=fa

[21] Fadaei M. [The relationship between lifestyle and the meaning of life in Alfred Adler's thought (Persian)]. J Res Bull Lifestyle. 2016; 2(3):65-76. https://www.magiran.com/paper/1658090

[22] Ghanbari Sartang A, Dehghan H, Abbaspour Darbandi A. [A review of health promotion lifestyle comparisons in shift and non-shift nurses (Persian)]. Iran J Nurs Res. 2015; 2(2):32-8. http://ijrn.ir/article1-195-fa.html

[23] Atadakht A, Rahimi S, Vali Nejad S. [The role of lifestyle enhancing health and religious orientation in predicting quality of life and death anxiety in the elderly (Persian)]. Aging Psychol. 2018; 4(2):143-54. https://jap.razi.ac.ir/article_1002.htm

[24] Mousavi M, Moadab N, Jafari A, Lael-Monfared E. The relationship between health restraint and health-promoting behaviors and quality of life of students in Torbat-e Heydarieh city in 2017-2018. Journal of Torbat Heydariyeh University of Medical Sciences Student Research Committee. 2019; 1(1):2-6. http://jms.thums.ac.ir/en

[25] Walker SN, Hill-Polerecky DM. Psychometric evaluation of Health Promoting Lifestyle Profil II. Unpublished manuscript, University of Nebras ka Medical Center; 1996. https://deepblue.lib.umich.edu/bitstream/ handle/2027.42/85349/HPLP_IIDimensions.pdf?sequence=2

[26] Basharpoor S, Heydarirad H, Atadokht A, Daryadel SJ, Nasiri-Razi R. [Health-promoting lifestyle:The role of beliefs and lifestyle-promoting lifestyle in predicting anxiety in pregnant women (Persian)]. Iran J Health Educ Health Promot. 2015; 3(3):171-80. http://journal.ihepsa. ir/article-1-260-fa.htm

[27] Salimi R, Jalili E, Advai N, Sanaei Z. [CT scan findings in children referred to the emergency department with head trauma; A cross-sectional 
study (Persian)]. Iranian Journal of Emergency Medicine. 2020; 7(1). https://journals.sbmu.ac.ir/iranjem/article/view/25905/19481

[28] Ryff CD. Happiness is everything, or is it Explorations on the meaning of psychological well-being. J Pers Soc Psychol. 1989; 57(6):1069-81. [DOI:10.1037/0022-3514.57.6.1069]

[29] Chloe Uyen H. Promoting mental health in older adults [PhD. dissertation]. Lubbock: Texas Teach University; 2009. http://hdl.handle. net/2346/16631

[30] Bayani AA, Kouchaki AM, Bayani A. [Narrative and reliability of the scale to Rif's psychological biology (Persian)]. Iran J Psychiatry Clin Psychol. 2008; 14(2):146-51. http://ijpcp.iums.ac.ir/article-1-464-en. html

[31] Jiang W, Kuchibhatla M, Cuffe MS, Christopher EJ, Alexander JD, Clary GL, et al. Prognostic value of anxiety and depression in patients with chronic heart failure. Circulation. 2004; 110(22):3452-6. [DOI:10.1161/01.CIR.0000148138.25157.F9] [PMID]

[32] Sadoughi M, Hesampour F. [Predicting the psychological well-being of Kashan University of medical sciences students based on the components of forgiveness (Persian)]. Iran J Neonatol. 2018; 30(110):1-10. [DOI:10.29252/ijn.30.110.1]

[33] Fatemi MM, Zare M, Kharrazi Afra F, Kharrazi Afra M. [Investigating the relationship between spiritual growth and how to deal with stressful conditions among medical interns in Islamic Azad University, Tehran Branch (Persian)]. Med Sci. 2014; 24(1):49-53. http://tmuj.iautmu. ac.ir/article-1-772-fa.html 